\section{Kombinierte Therapie}

E in wichtiges Therapieziel bei Rhinosinusitis ist neben der Sekretolyse die Eindämmung der überschießenden Entzündungsreaktion. Sinupret ${ }^{\circledR}$ besitzt eine breit angelegte antiinflammatorische Wirkung über die Inhibition verschiedener Entzündungsmediatoren des Immunsystems. Neue Daten zeigen, dass das Phytotherapeutikum beispielsweise die Enzyme Cyclooxygenase-2 und 5-Lipoxygenase hemmt. Beide sind wichtige Faktoren bei der Produktion von Prostaglandinen bzw. Leukotrienen. Sinupret ${ }^{\circledR}$ hemmt die Immunreaktion nicht vollständig, sondern dämmt lediglich die überschießende Entzündungsreaktion ein.

Die antiinflammatorische Wirkweise ergänzt die sekretolytische Wirkung von Sinupret ${ }^{\circledR}$. Beides zusammen unterstützt die Wiederherstellung von Ventilation und Drainage und die mukoziliäre Clearance kann das zähe Sekret mitsamt Pathogenen wieder abtransportieren.

Für die Wirksamkeit von Sinupret ${ }^{\circledR}$ sind vor allem Flavonoide verantwortlich. Sie sind die häufigsten sekundären Pflanzeninhaltsstoffe und kommen in vielen Früchten, aber auch in Gemüsesorten oder Rotwein vor. Durch seine antiviralen und antibakteriellen Eigenschaften wirkt Sinupret ${ }^{\circledR}$ nicht nur den äußerst unangenehmen Symptomen der Rhinosinusitis entgegen, sondern bekämpft gleichzeitig auch die Ursachen der Erkrankung.

Nach Informationen von Bionorica, Neumarkt i. d. Oberpfalz

\title{
Saisonale Asthma-Exazerbationsrate deutlich gesenkt
}

$\mathrm{N}$ ach aktuellen Studienergebnissen kann der monoklonale IgE-Antikörper Omalizumab (Xolair®) die saisonale Erhöhung der Zahl der Exazerbationen bei Kindern und Jugendlichen mit schwerem allergischen Asthma nahezu vollständig verhindern. Dies geht aus einer Post-hoc-Analyse der ICATAStudie hervor. An dieser doppelblinden, placebokontrollierten Untersuchung hatten 419 Patienten im Alter von sechs bis 20 Jahren aus innerstädtischen Gebieten in den USA teilgenommen. Die Exazerbationsraten im Frühjahr und im Herbst („back-to-school-Asthma“) blieben unter Omalizumab mit 4,3 und $4,2 \%$ gegenüber dem Sommer mit $3,3 \%$ nahezu konstant. Dagegen war in der Placebogruppe mit 9,0 und 8,1\% im Frühjahr und Herbst gegenüber dem Sommer $(4,6 \%)$ etwa eine Verdoppelung nachweisbar.

„Dass Omalizumab bei Kindern und Jugendlichen insbesondere auch die Zunahme der Exazerbationen zu Schulbeginn im September fast komplett verhindern kann, stellt eine wich- tige neue Erkenntnis für die Behandlung dieser Patienten dar", unterstrich Prof. Dr. Eckard Hamelmann, Bochum. $\mathrm{Da}$ in Städten eine besonders hohe Exposition gegenüber Allergenen vorliegt, sind gerade die dort lebenden Patienten vom saisonalen allergischen Asthma betroffen. Die allergene und infektiöse Belastung der Schüler durch den Schulbesuch ist nach den Sommerferien bzw. Urlaub deutlich erhöht.

„Damit verbunden sind massive Einschränkungen asthmatischer Kinder, die häufig gleich zu Beginn des neuen Schuljahres am Unterricht gehindert werden", so Hamelmann. Mit Omalizumab könnten nach den neuen Ergebnissen diese saisonalen Exazerbationsspitzen gesenkt und dadurch Fehlzeiten verhindert werden.

red

Nach Informationen von Novartis Pharma, Nürnberg
Hautarztverfahren - so geht's

Das Hautarztverfahren soll dazu beitragen, beruflich bedingte Dermatosen möglichst frühzeitig zu erkennen. Eine Kombination aus Therapie- und Präventionsmaßnahmen soll dabei eine Chronifizierung der Erkrankung und somit eine Berufsunfähigkeit verhindern. Obwohl das Verfahren für Patienten, Ärzte und Kostenträger nützlich sein kann, wird noch zu wenig davon Gebrauch gemacht. Vor diesem Hintergrund fand Anfang Mai 2011 in München ein vom Unternehmen Astellas unterstütztes, interdisziplinäres Seminar statt, das über die dermatologischen und verwaltungsrechtlichen Modalitäten des Hautarztverfahrens aufklärte. Dabei wurde betont, dass die für das Hautarztverfahren notwendigen diagnostischen Leistungen ohne Genehmigung der Unfallversicherungsträger durchgeführt und abgerechnet werden können.

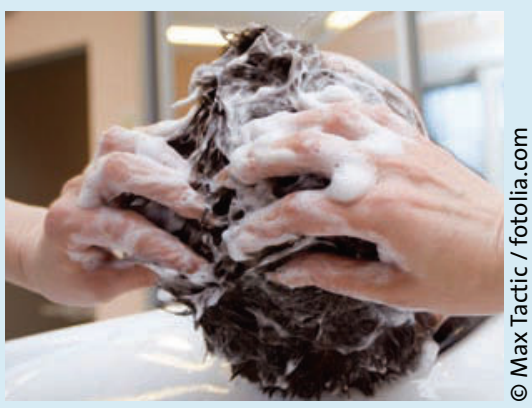

Weitere Seminartermine sowie eine Broschüre und ein Merkblatt zum Hautarztverfahren können unter der Tel.-Nr.: (o 89) $4544-1477$ abgerufen werden. Die nötigen Formblätter für das Hautarztverfahren stehen im Internet unter www.dguv. de/formtexte/aerzte/index.jsp zur Verfügung.

Nach Informationen von Astellas, München

\section{Fluticasonfuroat zum Festbetrag}

Im Juli 2011 hat der Arzneimittelhersteller GlaxoSmithKline den Preis von Avamys ${ }^{\circledR}$ um $18 \%$ und damit auf Festbetragsniveau gesenkt. Das Präparat mit dem Wirkstoff Fluticasonfuroat eignet sich zur Behandlung der saisonalen wie auch der perennialen allergischen Rhinitis. Hier reduziert es signifikant alle Nasensymptome und wirkt stark auf die Augensymptome. Die Preissenkung entlastet das Budget des behandelnden Allergologen.

Nach Informationen von GlaxoSmithKline, München 